\title{
Nature Inspired Online Real Risk Assessment Models for Security Systems
}

\author{
Ajith Abraham \\ Norwegian Center of Excellence \\ Norwegian University of Science and Technology \\ O.S. Bragstads plass 2E, N-7491 Trondheim, Norway \\ ajith.abraham@ieee.org \\ http://www. softcomputing.net
}

Very often, risk assessment in security systems is often done by human experts, because there is no exact and mathematical solution to the problem. Usually the human reasoning and perception process cannot be expressed precisely. Different people have different opinions about risk and the association of its dependent variables. We first present the role of fuzzy inference methods to develop intelligent online risk assessment models. We further illustrate the optimization of fuzzy inference systems using neural learning and evolutionary learning for using such models in an online environment. All the developed models are used in an intrusion detection/prevention system for online risk assessment. Finally, we present genetic programming models that could combine both intrusion detection and risk assessment and easily deployed in a mobile environment.

\section{References}

[1] Abraham, A., Jain, R., Thomas, J., Han, S.Y.: D-SCIDS: Distributed Soft Computing Intrusion Detection Systems. Journal of Network and Computer Applications 30(1), 81-98 (2007)

[2] Abraham, A., Grosan, C., Martin-Vide, C.: Evolutionary Design of Intrusion Detection Programs. International Journal of Network Security 4(3), 328-339 (2007)

[3] Haslum, K., Abraham, A., Knapskog, S.: HiNFRA: Hierarchical Neuro-Fuzzy Learning for Online Risk Assessment. In: Second Asia International Conference on Modeling and Simulation, AMS 2008, pp. 631-636. IEEE Computer Society Press, Los Alamitos (2008)

[4] Haslum, K., Abraham, A., Knapskog, S.: Fuzzy Online Risk Assessment for Distributed Intrusion Prediction and Prevention Systems. In: Tenth International Conference on Computer Modeling and Simulation, UKSiM/EUROSiM 2008, pp. 216223. IEEE Computer Society Press, Cambridge (2008)

[5] Haslum, K., Abraham, A., Knapskog, S.: DIPS: A Framework for Distributed Intrusion Prediction and Prevention Using Hidden Markov Models and Online Fuzzy Risk Assessment. In: Third International Symposium on Information Assurance and Security, pp. 183-188. IEEE Computer Society press, Los Alamitos (2007) 